\title{
Reflexiones acerca del Antropoceno
}

\author{
FRANZ MAUELSHAGEN
}

1

Reflections about Anthropocene

FRANZ MAUELSHAGEN

Institute for Advanced Sustainability Studies,

Potsdam, Alemania

franz.mauelshagen@iass-potsdam.de

Traducción: Amanda Sucar Warrener

Desacatos 54, mayo-agosto 2017, pp. 74-89 a primera vez que me encontré con el término Antropoceno fue

en 2008, mientras preparaba una serie de conferencias universitarias sobre la historia climática de los siglos XIX y XX. El aumento de las temperaturas marca un profundo corte en la historia climática y revierte la tendencia al enfriamiento de la Pequeña Edad de Hielo (PEH) anterior. En la búsqueda de un marcador descriptivo del corte que el calentamiento global antropogénico representa en la historia climática de la Tierra, me encontré con una serie de artículos en los que se sugería el término Antropoceno. No había mucho en 2008. A pesar de lo llamativo de la idea detrás del concepto, desde el principio tuve problemas con òv $\theta \rho \circ \pi$ o — anthropos - La mayoría de las contribuciones al debate sobre el Antropoceno fueron escritas por científicos naturales, quienes, implícita o explícitamente, aplicaron un concepto de la especie del anthropos, lo humano, algo que investigadores que trabajan en ciencias sociales y humanidades encuentran alienante. También resulta enajenante para ellos ver que la capacidad de cognición a menudo se considera la característica más sobresaliente de nuestra especie, homo sapiens sapiens, lo que supuestamente ayuda a explicar el enorme potencial de transformación que se ha desplegado en su relativamente breve historia como especie biológica. Estas concepciones del ser humano se combinan a menudo con una especie de realismo científico que los investigadores que trabajan en estudios de la ciencia y la historia de la ciencia han estado ansiosos por deconstruir una y otra vez durante los últimos 30 y tantos años. Esto ya es una clara indicación de que, en el debate sobre el Antropoceno, dos estilos académicos están chocando.

Una de las críticas más frecuentes y serias del término Antropoceno ha sido que cobija partes desiguales en el establecimiento del 
cambio ambiental global bajo la sombrilla de "lo humano", un asunto al que todos los artículos de este número se refieren de una u otra forma. Volveré a esta cuestión de la desigualdad en la siguiente sección. En este punto, simplemente me refiero a ella como una ilustración del problema referente a los estudios que explican la idea del Antropoceno desde el punto de vista de las ciencias naturales. En particular, algunos de los primeros que se publicaron parecen negar la mayor parte del conocimiento general que ha surgido en las últimas tres décadas dentro de las humanidades y las ciencias sociales con enfoque cultural. Desde el punto de vista de éstas, proponer el término Antropoceno puede parecer inapropiado y surgido de la ignorancia. Sin embargo, la ignorancia, entre las ciencias naturales - $\mathrm{O}$ en nuestro caso, más precisamente, los científicos de la Tierra involucrados en la comprensión del cambio global-y las ciencias sociales/humanidades, es mutua, y por lo tanto — ide nuevo! —, es en gran parte una cuestión de perspectiva. Necesitamos ver y reflexionar dónde nos encontramos como individuos e investigadores con cierta experiencia, no sólo apuntar a los que están del otro lado. La ignorancia entre las "dos culturas", descrita por C. P. Snow (1959) tan acertadamente hace casi 60 años, no es ninguna novedad. ${ }^{1}$ La noticia es que el Antropoceno es un concepto que nos desafía, nos pide que reduzcamos la brecha y crucemos. Pero también hay que tener cuidado, ya que enfrentar ese desafío tiene el potencial de una experiencia de tipo cesárea: una vez que se cruza el Rubicón, no hay vuelta atrás.

\section{2}

Mucho se ha escrito sobre el Antropoceno desde que me encontré con el concepto por primera vez en 2008. Las humanidades y las ciencias sociales se han vuelto más y más sensibles a él y se han vertido algunas críticas. De hecho, muchos investigadores son muy críticos al respecto y cuestionan sus propias bases. Este enfoque implica el peligro de reducir el debate sobre el Antropoceno a una disputa alrededor de una palabra, en la que el sentido parece fallar debido a que no se encuentra un lenguaje común. Ese lenguaje no puede ser inventado. Hay que dejar que emerja como parte de nuestras reuniones interdisciplinarias y sus resultados impredecibles. A estas alturas parece como si los académicos no se hubiesen congregado en este tipo de reuniones durante siglos, y ahora, cuando se reúnen, después de un largo periodo de espléndido aislamiento disciplinario, asemejan una asamblea de jefes que no están familiarizados con el idioma del otro. En la actualidad, el número de traductores — aquellos educados en ambas lenguas o campos científicos- es insuficiente debido a las prácticas establecidas desde hace tiempo en nuestros sistemas académicos para estudiar $y a$ las ciencias naturales $o$ las ciencias sociales y las humanidades.

El experimento del diálogo interdisciplinario se ejerce actualmente en el Grupo de Trabajo del Antropoceno (AWG, por sus siglas en inglés) establecido por la Comisión Internacional de Estratigrafía en 2009. En ocasiones es malinterpretado como un grupo de geólogos o científicos naturales que buscan arrebatar de nuestras manos la definición de una nueva época geológica, en lugar de dejarla abierta al discurso, tanto crítico como afirmativo, y a la pluralidad de perspectivas disciplinarias. A veces, el AWG es considerado incluso otro ejemplo para el dominio de las ciencias naturales sobre el resto de la academia. Sin embargo, sus miembros provienen de diversos orígenes disciplinarios. Naturalmente, aportan una gran cantidad de conocimientos derivados de las ciencias de la Tierra, pero, al final, la recomendación del AWG estará obligada

1 Kagan (2009) consideró más apropiado reconocer las fronteras entre las ciencias sociales y las humanidades, y por consiguiente, referirse a tres culturas. 
a aplicar reglas establecidas para identificar los periodos geológicos en el registro estratigráfico. Estas reglas fueron diseñadas para cumplir con normas disciplinarias muy específicas. Sin embargo, Jan Zalasiewicz, coordinador del AWG, ha invitado a un importante número de expertos provenientes de las humanidades y ciencias sociales. ${ }^{2}$

El 29 de agosto de 2016, en el Congreso Internacional de Geología celebrado en Ciudad del Cabo, Sudáfrica, el AWG presentó su recomendación para formalizarlo. Sin embargo, es poco probable que debido a ello el debate interdisciplinario se haya detenido. Lo que ciertamente constituye un triunfo para Eugene Stoermer, Paul Crutzen y sus seguidores puede ser visto como un malentendido desastroso desde el punto de vista de los demás. Tomando partido en esta controversia, me cuento entre los que apoyan la idea de Crutzen. Sin embargo, mi afirmación está acompañada por el escepticismo respecto a un grupo importante de científicos que acogen la idea del Antropoceno porque, a sus ojos, añade legitimidad al dar el siguiente paso evolutivo en la historia del control científico sobre la "naturaleza" y posiblemente también sobre las sociedades humanas y su futuro. ${ }^{3}$

El AWG consideró varias fechas potenciales del comienzo del Antropoceno. Todas tienen implicaciones para la trayectoria histórica del cambio global, así como el nivel y el alcance geográfico de la acción humana. Paul Crutzen y Eugene Stoermer habían sugerido el principio a partir de la industrialización temprana: Inglaterra a finales del siglo XVIII (Crutzen, 2002a; 2002b, Crutzen y Stoermer, 2000; Steffen, Crutzen y McNeill, 2007; Steffen et al., 2011). Esa fecha fue dominante hasta muy recientemente. Si bien se han sugerido otras fechas de comienzo muy anteriores, como menciona Helmuth Trischler en este número de Desacatos, las cuales resultan valiosas porque reconocen la importancia de los cambios ambientales antropogénicos previos al periodo industrial, Crutzen, Steffen,
McNeill y Grinevald han argumentado de manera convincente contra tales intentos. También han sugerido una cronología más sofisticada para el Antropoceno. Después de la industrialización temprana en Europa y Estados Unidos hubo un periodo de crecimiento económico acelerado, cuyos principales efectos sobre el sistema terrestre se presentaron a partir de ca. 1950. Este segundo periodo se ha denominado la "gran aceleración” (Steffen et al., 2015). En los últimos dos o tres años, la tendencia ha sido cambiar la fecha de comienzo del Antropoceno de aquélla de la industrialización temprana a la de la gran aceleración (Lewis y Maslin, 2015; Waters et al., 2016; Zalasiewicz et al., 2015). Precisamente esto es lo que el AWg propuso en agosto de 2016.

A pesar del poco interés que los historiadores tienen en la estratigrafía, favorecer la gran aceleración durante el industrialismo temprano en Europa también tiene mucho sentido desde el punto de vista de la historia global y el poscolonialismo. Como ha sido el caso con la palabra "antropogénico" en "cambio climático antropogénico", el término Antropoceno ha sido criticado por disfrazar partes desiguales en la creación de la crisis ambiental global, y por lo tanto, su responsabilidad detrás del extenso concepto de "actividades humanas" en el sistema terrestre. Los indicadores económicos, así como las emisiones de gases de efecto invernadero sugieren que la idea de una humanidad que comparte el patrón industrial de crecimiento basado en

2 La lista de miembros del AWG aparece en la página web de la Subcommission of Quaternary Stratigraphy. Disponible en línea: <http://quaternary.stratigraphy.org/workinggroups/ anthropocene/>. Consultado el 3 de septiembre de 2016. El propio trabajo de Zalasiewicz da testimonio de la multidimensionalidad y la multidisciplinariedad necesarias para evaluar el Antropoceno (Zalasiewicz, 2010; Zalasiewicz et al., 2010; 2015; Zalasiewicz y Williams, 2011).

3 La perspectiva ecomodernista puede servir como ejemplo de esto, en particular el Breakthrough Institute (Shellenberger y Nordhaus, 2015). Más cauteloso es Ellis (2015). 

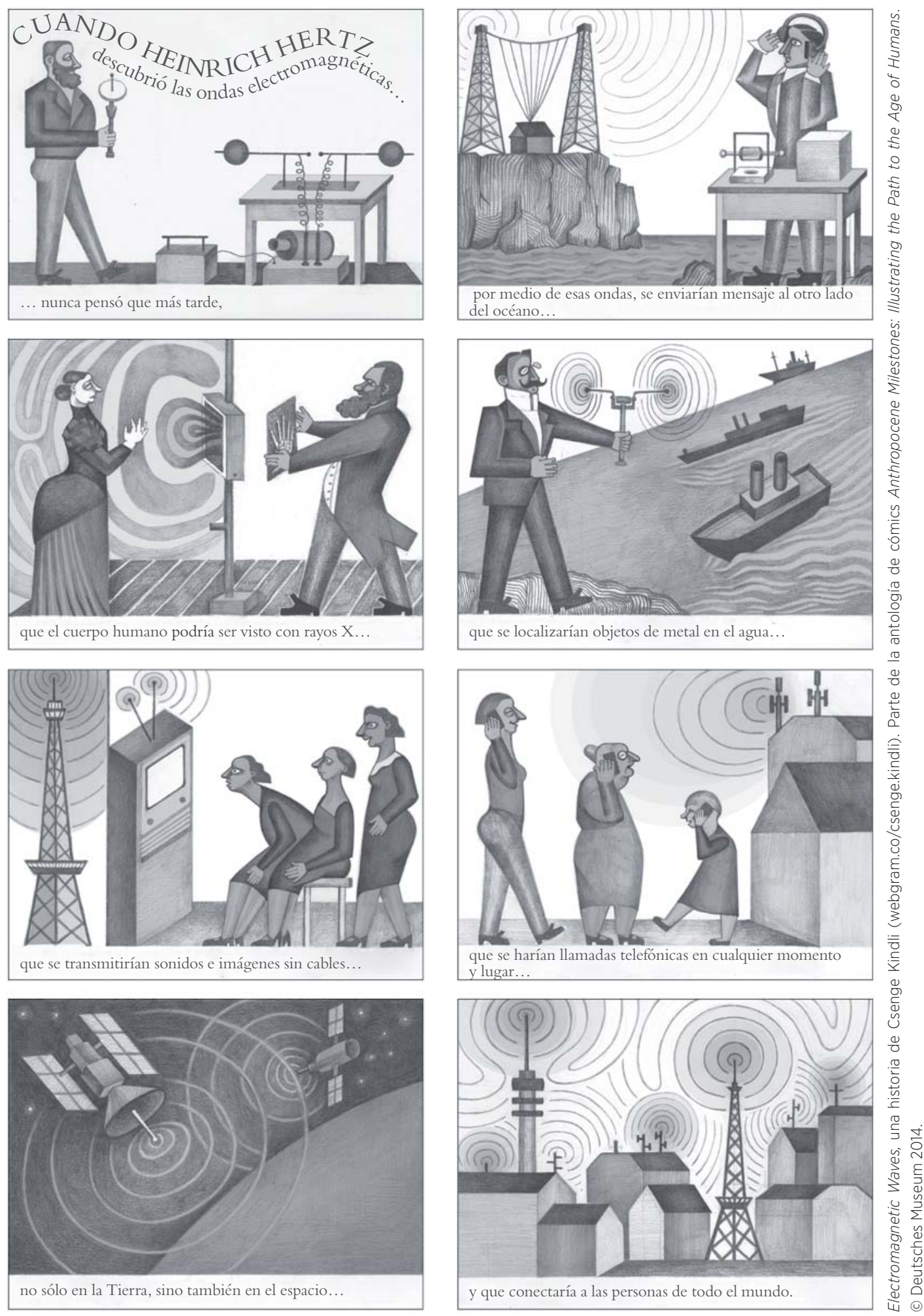
Consumo de energía primaria.

Equivalente a millones de toneladas de petróleo
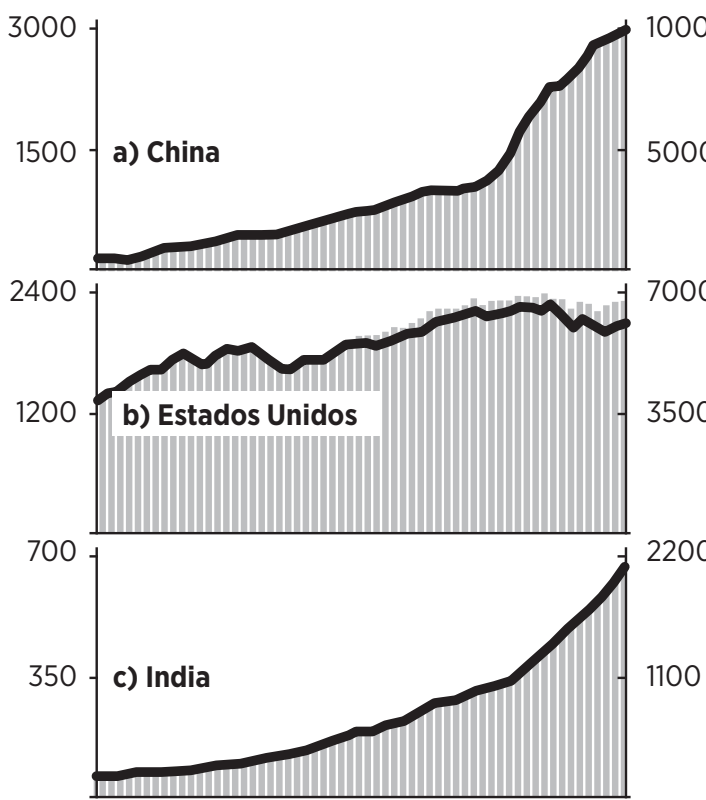

230

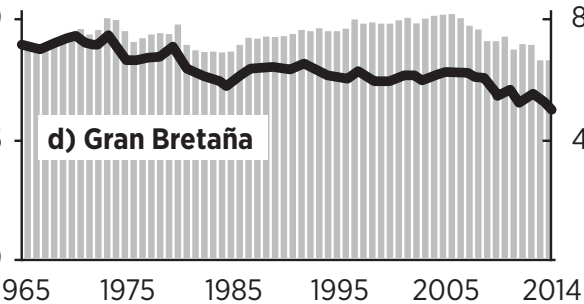

400
Consumo de energía por sector. Equivalente a millones de toneladas de petróleo

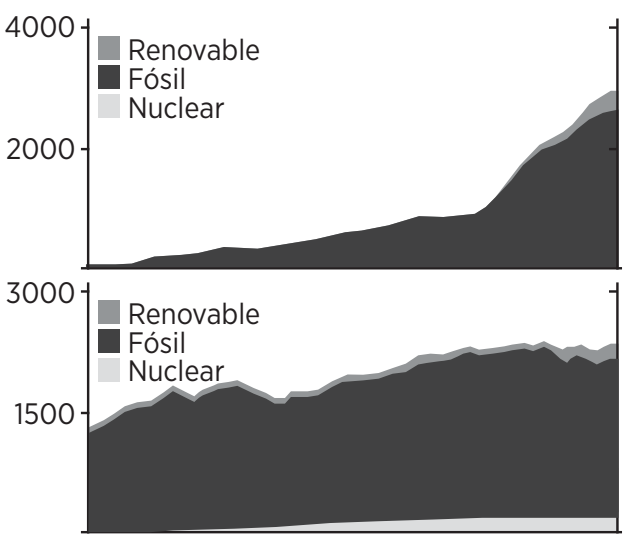

2200

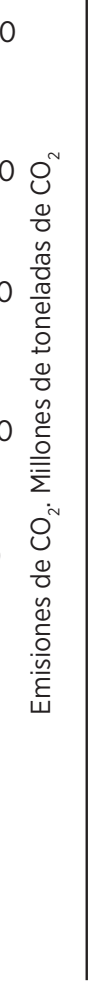

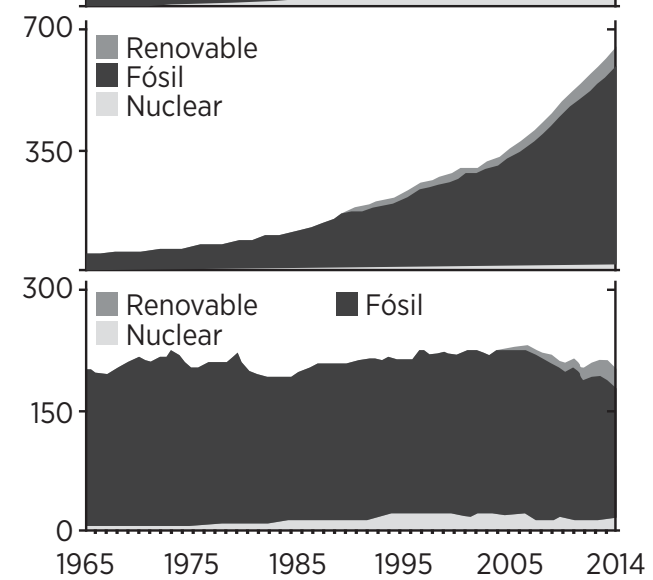

Figura 1. Consumo de energía primaria y emisiones de $\mathrm{CO}_{2}$ (izquierda), y consumo de de energía mixta para China, Estados Unidos, India y Gran Bretaña (derecha), 1965-2014

el consumo de energía fósil, establecido por primera vez en Europa y Norteamérica, se materializa sólo después de la Segunda Guerra Mundial: primero lentamente y luego cada vez más rápido (véase la figura 1). Mientras que las desigualdades en el consumo de energía per cápita y las emisiones de gases de efecto invernadero siguen siendo significativas hoy, el fin del colonialismo permitió el ascenso de las economías industriales, sobre todo en Asia. ${ }^{4}$ Últimamente, las naciones BRICS - China más que los otros países - ${ }^{5}$ le dieron a la gran aceleración su último impulso. Sólo a partir de la gran aceleración podemos hablar de una distribución verdaderamente global de los principales emisores. La elección, en cambio, de la industrialización temprana en Inglaterra como el punto de partida del Antropoceno implica claramente el peligro de caer en la vieja trampa del eurocentrismo. Esto es, entre otras razones, el porqué de que historiadores como Dipesh Chakrabarty y John McNeill prefieren la

Para una versión actualizada de las gráficas de aceleración cuyos datos en conjunto - datasets - permiten distinguir entre la Organización para la Cooperación y el Desarrollo Económicos (OCDE), BRICS - siglas para designar a Brasil, Rusia, India, China y Sudáfrica- y otros países, véase Steffen et al. (2015).

5 Veáse la nota 4 
gran aceleración como punto de partida en la cronología del Antropoceno. ${ }^{6}$

\section{3}

Todos los artículos de este número de Desacatos se refieren al papel del capitalismo en el proceso del Antropoceno, pero la contribución de Astrid Ulloa se ocupa de él con mayor extensión que los otros. No hay duda alguna de que la historia del capitalismo y su difusión mundial, a menudo por medio de las venas del colonialismo y el imperialismo, está profundamente involucrada con el cambio ambiental global. ${ }^{7}$ El problema de las economías extractivistas, un tema presente con fuerza en el discurso latinoamericano sobre cuestiones ambientales y las raíces coloniales del capitalismo, es casi universal en la actualidad. En el mundo moderno, desde los albores de la industrialización, las sociedades capitalistas han desarrollado la dinámica económica más grande de la historia humana, un periodo prolongado de crecimiento que ha incrementado y acelerado la producción de energía y bienes y servicios. Dondequiera que el capitalismo ha ganado un punto de apoyo, desde los centros de la industrialización temprana en Europa, hasta América, Japón, y hoy en día, India y China, ha dado lugar a las más severas huellas ecológicas per cápita.

Sin embargo, sostengo la idea de que hay que "llamar a las cosas por su nombre", y por lo tanto, prefiero el término Capitaloceno. ${ }^{8}$ Mientras que el papel del capitalismo como una fuerza impulsora del cambio global no puede ser ignorado, no veo ninguna ventaja en un término que corre el riesgo de la simplificación monocausal. Las raíces históricas del cambio ambiental antropogénico, incluso las del cambio climático antropogénico, van más allá de la historia del capitalismo industrial. ${ }^{9}$ Además, no podemos ignorar que durante el siglo XX grandes partes del mundo fueron dominadas por el comunismo industrial. Su legado ha dejado poca evidencia, si la hay, de que eran menos dañinos para el medio ambiente que las economías capitalistas liberales. Julia Adeney Thomas, en su artículo en este número de Desacatos, desarrolla un argumento más completo acerca del Capitaloceno. También hace hincapié en el papel del imperialismo, que históricamente se mezcla con el capitalismo, aunque están lejos de ser idénticos.

En octubre de 2015, tuve el privilegio de asistir a las dos últimas conferencias que el novelista e intelectual Amitav Ghosh (2016) ofreció en la Universidad de Chicago. ${ }^{10}$ Ghosh sostuvo que el Antropoceno está tan envuelto con la historia del imperio y el imperialismo como lo está con la del capitalismo. El capitalismo y su dominio en la era industrial dependían del poder imperial ejercido por los Estados europeos sobre Asia y otras partes del mundo. El control imperial protegía las industrias europeas y americanas del carbón y el petróleo del desarrollo de las mismas industrias en Asia antes de la descolonización, lo que explica la entrada tardía de India

6 Ésta es la cronología utilizada por McNeill y Engelke (2014). Chakrabarty $(2014 ;$ 2015) sostiene que la gran aceleración coincide con un periodo de creciente descolonización después de la Segunda Guerra Mundial.

7 Chakrabarty (2009; 2014) ha dejado muy claro que el clima y el capital tienen historias unidas - conjoined histories-.

8 Promovido por Moore (2014a; 2014b; 2016). Bonneuil y Fressoz (2016) son también partidarios de él. Para un estudio de algunos de los neologismos que ha inspirado la idea del Antropoceno, véase Haraway (2015).

9 Se trata de un consenso actual entre los historiadores ambientales y los arqueólogos. En las secciones históricas de dos artículos seminales de Steffen, Crutzen y McNeill (2007) y Steffen et al. (2011) se encuentran sendos resúmenes de la idea del Antropoceno y su cronología.

10 El ensayo de Gosh constituye la versión impresa, ligeramente actualizada, de sus impresionantes Berlin Family Lectures, presentadas en septiembre y octubre de 2015 en la Universidad de Chicago. Disponible en línea: <https:// berlinfamilylectures.uchicago.edu/2015-amitav-ghosh>. Consultado el 3 de septiembre de 2016. 
y China al club de las economías fósiles. Ghosh no duda en señalar la ambivalencia inherente al papel histórico que el imperialismo ha jugado en esa trayectoria: si bien ha creado desigualdades sin precedentes mediante el fomento de la industrialización en una parte del mundo y la ha obstaculizado en otra, desde el punto de vista del calentamiento global ese mismo imperialismo ha provocado un retraso en la gran aceleración (Ghosh, 2016: 109-110; Chakrabarty, 2014: 15). Por supuesto, no existe razón para ensalzar al imperialismo por provocar un efecto colateral involuntario. El imperialismo, de hecho, ha ganado algo de tiempo en el cambio climático antropogénico al negar a las economías colonizadas la misma prosperidad económica, es decir, que no se produjo un escenario alternativo en el que éstas hubieran empezado antes a tener economías basadas en emisiones de carbono. No obstante, también podría argumentarse que el imperialismo tiene, al mismo tiempo, patrones de desarrollo occidentales globalizados, y por lo tanto, impidió el surgimiento de una mayor variedad de futuros sociales respecto a cómo las sociedades organizan su consumo de energía y la producción de bienes y servicios. Por el contrario, la modernización fósil y su impulso imperial han unificado el mundo humano en todas las sociedades y culturas en su deseo de obtener mayor riqueza material. Mientras que las culturas alrededor del mundo parecen revelar una enorme variedad, esa misma diversidad se reduce drásticamente si se consideran las estrategias relacionadas con energía y recursos. Con ligeros matices, se muestran iguales en todas partes, sobre todo cuando se trata de compartir la prosperidad de la que gozan las naciones ricas. Esta homogeneización de las estrategias ecológicas básicas, teniendo el régimen de energía como uno de los determinantes primordiales en las relaciones hombre-ambiente, constituye un indicador de la consideración de que el desarrollo sostenible, como "desarrollo", implica compartir la prosperidad entre todos. ${ }^{11}$ También indica que el giro cultural ha creado probablemente un sesgo dentro de las humanidades y las ciencias sociales: un énfasis excesivo en las diferencias culturales en comparación con las semejanzas. Este sesgo sólo será superado por un cambio de paradigma en la teoría cultural que reconoce la dimensión ecológica en la evolución de las culturas. La integración de perspectivas históricas y arqueológicas, en otras palabras, perspectivas a más largo plazo de la ecología humana y cultural, facilitará dicho esfuerzo, sobre todo en las ciencias sociales, cuyo horizonte en ocasiones se estrecha al focalizar sólo las sociedades contemporáneas y modernas.

A lo largo de sus conferencias, Ghosh combinó el Antropoceno con la figura de reversión - reversal-: la reversión de la modernidad (2016: 21-22, 62-63, 80, 113, 121). El cambio climático es un signo de que el "inconcluso proyecto de la modernidad" - Habermas - está a punto de derrumbarse..$^{12}$ Las teorías de la modernidad rara vez consideraron esa posibilidad. Muy por el contrario, han apoyado las afirmaciones modernistas de la irreversibilidad casi sin excepción. Ghosh sostuvo profunda y poderosamente que la idea del Antropoceno cuestiona tales afirmaciones. La más fundamental de todas las reversiones es su cuestionamiento del control de la modernidad sobre y la independencia de la "naturaleza”. En su lugar, el Antropoceno requiere que (re)definamos el lugar de los seres humanos y sus sociedades dentro de la red de la vida y eso es precisamente el punto en el que definir el anthropos no sólo como un animal social, sino como una especie animal, es útil y puede ayudar a las ciencias sociales y

11 Para una discusión más elaborada alrededor de modernización y desarrollo sustentable, véase Mauelshagen (2015: 179-184).

12 Passerin d'Entrèves (1996: 38-55) ofrece una traducción al inglés del ensayo de Habermas, "Modernity: An Unfinished Project". 
humanidades a ampliar el horizonte y relativizar su comprensión de "lo humano".

No reconocernos como especie biológica, es decir, uno entre un sinnúmero de otros que viven en la biosfera, socava precisamente esos axiomas de las humanidades y las ciencias sociales que cortan las conexiones entre el mundo social y su entorno no humano, en particular la regla de Durkheim (1982: 134), de que "la causa determinante de un hecho social debe buscarse dentro de los hechos sociales que le antecedieron". ${ }^{13}$ Por supuesto, había una razón por la cual la inventó en primera instancia, ya que intentaba dejar atrás el determinismo natural de gran parte de la ciencia social temprana de su tiempo - basta pensar en su argumento en Le suicide, publicado en 1897, contra los supuestos determinantes climáticos que comúnmente se utilizaban para explicar la variabilidad en las estadísticas de suicidio respecto a la ubicación o el ciclo anual—. Sin embargo, más de un centenar de años después, la situación se ha invertido. La ciencia social y la teoría social prevalecientes excluyen el entorno por omisión. Éste es uno de los problemas más obvios relacionados con la división entre naturaleza y cultura que Bruno Latour (2005) y Philippe Descola (2013) se han comprometido a superar, y no deberíamos limitarnos a señalar a las ciencias naturales cuando abordamos este problema. De hecho, tanto Latour como Descola, en varios de sus libros, se han ocupado de la división y sus consecuencias dentro de sus propios campos de investigación. ${ }^{14}$ Sin embargo, muchos investigadores han seguido sus pasos y aceptan hablar de dientes para fuera sobre sus críticas, en lugar de aplicarlas a sus propias disciplinas. A lo que me refiero con "hablar de dientes para afuera" es a una práctica bastante común de reducir los problemas de sustentabilidad o conducta ambiental al dualismo cartesiano. El argumento recuerda al antiguo procedimiento "escolástico" de rastrear el origen de todos los pecados al pecado original de Adán. ¡Reduccionismo teórico espléndido! Pero, realmente, ¿qué tan confiable es la crítica que supone que hay una explicación universal —en nuestro caso, el dualismo cartesiano- para un problema complejo?

Para evitar el peligro del reduccionismo teórico, la división entre naturaleza y cultura debería sólo ser el punto de partida de nuestras consideraciones, no su resultado. Debemos tener cuidado con aquello de que se aplica mejor al sistema académico, en particular al diseñado en Occidente o a partir de él. ${ }^{15}$ Esta confesión puede ayudarnos a evitar proyectar abiertamente nuestra visión académica del mundo, o peor aún, la disciplinaria. No obstante, éste puede ser un primer paso para contribuir de manera significativa y concreta a entender las transformaciones que se encuentran en la base del cambio ambiental global y considerar qué se puede hacer para transformar nuestro mundo en un lugar más sustentable.

\section{4}

En su contribución para este número de Desacatos, Philippe Descola discute el problema de la apropiación, tan importante para nuestra comprensión de cómo los seres humanos toman posesión de su entorno y hacen uso de él. La comprensión de las diferencias culturales en la apropiación es una de las más valiosas contribuciones que los antropólogos pueden hacer al debate sobre el Antropoceno. Descola

\footnotetext{
13 Obra publicada originalmente en francés, en 1895.

14 Véase en particular Descola (2013). No puede pasarse por alto la polémica de Latour (2005) contra la sociología clásica. A pesar de su popularidad, en particular en el estudio de la ciencia y la historia de la ciencia, me parece que la recepción de las obras más recientes de Latour muestra que aún no hay un reconocimiento generalizado de lo que él ha denominado el environmental turn (Latour, 2013; 2015).

15 Ghosh (2016: 31, 64) hace interesantes comentarios alrededor del dualismo cartesiano. Véase también Mauelshagen (2015: 184-185).
} 


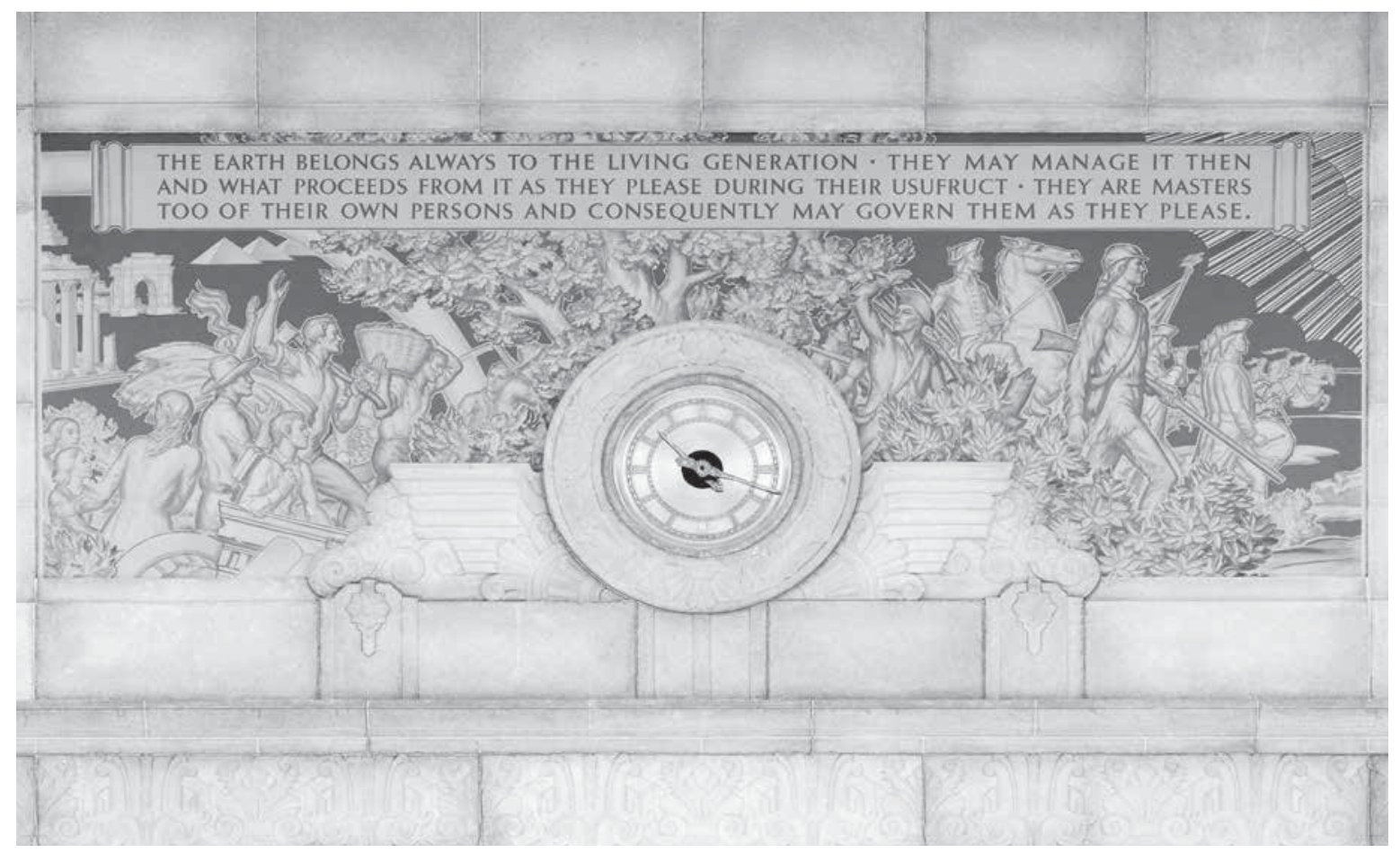

Figura 2. Mural de Ezra Winter con cita de Thomas Jefferson. Library of Congress, John Adams Building, South Reading Room, Washington, D. C.

habla de la tensión entre la propiedad privada y colectiva, y se refiere al famoso debate sobre la supuesta "tragedia de los comunes". No podría estar más de acuerdo con su afirmación de que el problema de los comunes no es la propiedad común como tal, sino cómo se definen los derechos para hacer uso de ella. En relación con el clima, la biodiversidad, la atmósfera, etc., y de manera más general, el ámbito de la interacción entre los seres humanos y estos "no humanos" cuya apropiación no puede ser privada sino sólo colectiva, él dice que los seres humanos sólo pueden reclamar el derecho de usufructo.

En 2011 y 2012 pasé varios meses investigando en la Biblioteca del Congreso en Washington, D. C. La mayoría de las veces me sentaba en la sala de lectura del sur del edificio John Adams, donde mi atención, de manera involuntaria, se centraba en uno de los "murales de Jefferson", que ilustran citas famosas de Thomas Jefferson (1743-1826), autor de la Declaración de Independencia y tercer presidente de Estados Unidos de América. En cuanto a estética, los murales no me parecieron particularmente atractivos. Pero una de las inscripciones me llamó la atención y no dejé de pensar en ella hasta que entendí por qué (véase la figura 2). La inscripción señala: "La tierra pertenece siempre a la generación viviente. Durante su usufructo, pueden administrarla, así como lo que de ella provenga, como les plazca. También son dueños de sus propias personas, y en consecuencia, pueden gobernarlas como les plazca”. ${ }^{16}$ Durante el tiempo que trabajé en la Biblioteca del Congreso, comencé a interesarme en cuestiones

16

"The earth belongs always to the living generation. They may manage it then and what proceedes from it as they please during their usufruct. They are masters too of their own persons and consequently may govern them as they please". 
de justicia intergeneracional y sustentabilidad, en especial en el contexto del cambio climático, lo que explica en cierta medida por qué me llamó la atención la referencia de Jefferson a la generación viviente y su derecho de autogobierno. En un primer momento, abogar por una propiedad de la tierra de la generación viviente me pareció como tomar una posición enfocada en el presente que simplemente ignora el problema de la justicia intergeneracional. Sin embargo, lo que me molestaba era la palabra "usufructo", una palabra heredada del derecho civil romano que se refiere al derecho de uso —usus - de una propiedad y el disfrute de sus frutos - fructuspor un tiempo limitado, normalmente acotado por la vida de una persona. Esta limitación de propiedad de la tierra ya está implícita en la idea de una generación viviente: el derecho de esa generación a utilizar su propiedad está obviamente limitada por la muerte y el derecho de autogobierno de la siguiente generación. Por lo tanto, la palabra "usufructo" indica claramente los límites en el derecho de apropiación de la tierra. El derecho de usufructo se pasa de una generación a la siguiente. En otras palabras, Jefferson ciertamente se ocupa de la justicia intergeneracional aquí. Pero, ¿dice algo que sea relevante para la sustentabilidad?

Obviamente, la cita de Jefferson exige una mayor contextualización. Así que comencé a investigar y me encontré con una frase en la obra de un clásico del medio ambiente, George Perkins Marsh, que sonaba como respuesta o continuación del pensamiento de Jefferson. En The Earth as Modified by Human Action, Marsh escribió: "el hombre olvidó hace demasiado tiempo que la tierra fue dada a él por usufructo únicamente, no para el consumo, y menos aún para el desperdicio derrochador" (1877: 33). Aquí "consumo" es lo opuesto a "usufructo". En otras palabras, si hacemos uso de los recursos proporcionados por la tierra para nuestra vida de manera que se consuman —o peor, que se consuman de manera que contaminen la tierra-, estamos violando el principio de usufructo. Si consumimos no sólo las frutas, sino también la fuente que las produce, no quedará fruta ni fuente para la próxima generación viviente. Eso es precisamente lo que significaba la limitación de los derechos de propiedad en el derecho civil romano, sobre todo en el contexto de la producción agrícola. El usufructuario, es decir, la persona a quien se le ha dado el derecho de usufructo, tiene derecho a consumir los frutos o cualquier producto renovable de una propiedad. Sin embargo, el derecho del propietario sobre esa propiedad habría sido violado si el usufructuario destruyera la fuente de producción, la tierra en particular. Por lo tanto, el usufructo implica que es obligación del usufructuario mantener la productividad de la tierra. Esa continuación está conectada directamente a la idea de sustentabilidad. Usufructo significa que el consumo se limita a los recursos renovables y esa forma de poseer la tierra es sustentable siempre y cuando se mantenga la renovabilidad — renewability-.

La inscripción es una cita de una carta que Jefferson escribió desde París a James Madison, el 26 de septiembre de 1789, sólo un par de meses después del estallido de la Revolución francesa. ${ }^{17}$ Su carta no trata directamente de la sustentabilidad en la gestión ambiental. Jefferson analizaba una cuestión distinta: "si una generación de hombres tiene derecho a obligar a otra", una cuestión de implicaciones significativas para el "gobierno" — lo que actualmente llamaríamos "gobernabilidad"-, como señaló desde el principio. Él construyó su argumento a partir del principio de "que la tierra pertenece en usufructo a los vivos", lo que él consideraba evidente por sí mismo. Con base en ello, Jefferson no vio ningún derecho natural para la generación viviente de obligar a la siguiente generación por medio de 17 De 1785 a 1789, Jefferson fue embajador de Estados Unidos
en Francia. 
la deuda o la ley: "ninguna sociedad puede hacer una constitución perpetua, o incluso una ley perpetua”, escribió. Con base en los cálculos de esperanza de vida de los registros de mortalidad y otros criterios —edad de madurez, etc.—, sugirió un lapso máximo de 19 años para las leyes y estaba dispuesto a aplicar este principio incluso a la Constitución de Estados Unidos, que acababa de ser ratificada el año anterior, 1788. También quería que la deuda del Estado se liquidara después de 19 años y que su monto fuese limitado por la capacidad de una sociedad de solventarla en ese tiempo. A su juicio:

\begin{abstract}
Ello pondría a los prestamistas, así como a los prestatarios, en guardia. Además, al reducir la capacidad de tomar prestado a sus límites naturales [es decir, sucesión generacional], pondría coto al espíritu de guerra, a las que había dado rienda suelta la desatención de los prestamistas de dinero a esta ley natural, según la cual generaciones sucesivas no son responsables de las que las preceden (Jefferson, 2016).
\end{abstract}

Por supuesto, los números sobre los que Jefferson basó sus cálculos no serían lo mismo hoy para Estados Unidos ni para Francia. Una desventaja obvia de aplicar su método es que la esperanza de vida desigual se traduciría directamente en la credibilidad financiera de una nación. Sin embargo, sus reflexiones sobre la justicia intergeneracional son sorprendentes y profundas. El usufructo de la tierra como un derecho colectivo de una sociedad significaba para él "que las generaciones venideras no son responsables de las que las preceden". Lo que es aun más notable, la misma idea suponía responsabilidad de la generación precedente para la siguiente. La falta de derecho a obligar a la generación siguiente por ley o deuda se definía por un límite al derecho de usufructo ejercido por la generación viviente.

¿Con qué contribuiría el "principio de usufructo generacional de Jefferson", como me atrevo a llamarlo aquí, a los problemas actuales de sustentabilidad? Por fascinante que parezca, esta cuestión es obviamente demasiado compleja para responderse en pocas palabras. Para ilustrar el problema basta pensar en los combustibles fósiles, la principal fuente de energía del mundo de hoy: ¿acaso los combustibles fósiles son parte de los frutos de la tierra que una generación, o varias generaciones sucesivas, tienen derecho a consumir? ¿O no? ¿Y qué hay de la atmósfera como un recurso que cambia de manera significativa y peligrosamente por la exhaustiva quema de combustibles fósiles? ¿Deberíamos tener en cuenta los procesos geológicos del petróleo crudo y los enormes lapsos de la generación de carbón?

Duraciones de cientos de millones de años no sólo exceden periodos de vida generacionales por varios órdenes de magnitud, incluso dejan obsoleta la existencia de nuestra especie, el homo sapiens sapiens, y sus parientes biológicos —otras especies de homo, homínidos, los monos, incluso mamíferosen la tierra. Esto ilustra el tipo de rompecabezas de sustentabilidad del que el Antropoceno nos hace conscientes. El mundo de Jefferson fue de tiempos agrícolas. La agricultura seguía siendo el campo de juego principal de la productividad humana, impulsada por preocupaciones sobre ciclos anuales de reproducción, complementada con más preocupaciones a largo plazo por el desgaste y erosión del suelo, etc. Mientras más han cavado en la tierra las civilizaciones modernas, más han interferido con el tiempo profundo y los procesos bio-geoquímicos en una escala que está más allá de cualquier rango familiar de control por parte de "la generación viviente", y menos confiables son nuestras percepciones del tiempo y el momento, que dependen en parte de nuestra naturaleza como especie y en parte de nuestra adaptabilidad al mundo sociocultural en el que vivimos. ¿Hay alguna posibilidad de que aprendamos a manejar este problema?

El principio de usufructo generacional de Jefferson puede no ser la respuesta, pero permite 
reflexionar; aunque sólo ofrece un caso excepcional para el pensamiento temprano sobre las relaciones intergeneracionales definidas por la responsabilidad asimétrica. Traducir las preguntas sobre el futuro de la sustentabilidad a los problemas de justicia generacional es una manera de integrar el mundo no humano al mundo social, y por lo tanto, un paso hacia la superación de la dependencia de la trayectoria de la separación entre naturaleza y cultura tan visible en nuestras tradiciones científicas y en la gobernanza de las sociedades modernas. Implica cuestiones de libertad individual y sus límites; los derechos y las relaciones de las instituciones colectivas, como las naciones, y su derecho a la autodeterminación. En otras palabras, me parece obvio que el cambio global plantee el reto de redefinir de manera sustancial nuestra libertad individual y colectiva.

El caso de Jefferson también puede ayudar a cuestionar la oposición entre derechos de propiedad privada y colectiva en la apropiación de la tierra y sus recursos, y qué tan estricta es realmente esa oposición en diferentes sistemas económicos. La distinción entre "ley natural" y "derecho positivo", que subyace al argumento de Jefferson, era típica de su tiempo. Por artificial que parezca hoy, este modo de pensamiento nos ayuda a discernir que cualquier regulación concreta de derechos de propiedad en nuestro sistema legal se basa en suposiciones acerca de la propiedad colectiva. No existe lo privado sin una forma colectiva de propiedad y la regulación legal de ambas siempre tiene implicaciones para la otra. Radicalizar la diferencia al abreviarla en una cuestión de elección entre "espíritu emprendedor individual/privado" versus "el Estado", o incluso "capitalismo" versus "comunismo", es peligrosamente reduccionista. La propiedad privada no puede existir fuera del colectivo y siempre está limitada en formas muy concretas por ese hecho.

También es importante tener esto en cuenta cuando evaluamos la participación de las diferentes formas de economía — que deben siempre, de una manera $\mathrm{u}$ otra, apropiarse de los recursos de la tierra para sus fines - en el proceso del Antropoceno. Para nosotros, Jefferson puede ser la versión de pensador liberal democrático y de libre mercado de finales del siglo XVIII y principios del XIX. Sin embargo, los demócratas liberales de ninguna manera tenían la idea de que los seres humanos son dueños de la tierra. En el caso de Jefferson, quizá tenía sus raíces en la tradición o fe cristiana, pero es más probable que se basara directamente en consideraciones filosóficas más abstractas de la "ley natural" típica de la Ilustración. Marx compartió la misma idea y concibió una transformación total del mundo natural por medio del esfuerzo humano - trabajoformación que al final neutralizaría la alienación entre la naturaleza y el mundo social. ${ }^{18}$

\section{5}

Por último, permítanme volver a las conferencias de Amitav Ghosh y su idea de que el Antropoceno es una "reversión" de la modernidad. ¿Cómo sería la reversión de la modernidad en términos más prácticos? ¿Qué es exactamente lo que debe revertirse, a menos que los políticos y las sociedades dejen su futuro en manos del destino? Por cierto, es muy probable que ese escenario termine en colapso: quizá el de la civilización occidental (Oreskes y Conway, 2014). De hecho, varios escenarios catastrofistas de la degradación del medio ambiente, el colapso social o la extinción de la especie humana han sido muy receptivos a la idea del Antropoceno (Cochet, 2015; Northcott, 2015; Semal, 2015). Sin embargo, el catastrofismo no es propio de la idea de Antropoceno.

18

Esta forma de pensar acerca de la transformación de la naturaleza es aún más fuerte en los Manuscritos económicos y filosóficos de Marx, de 1844 (2000: 279-400), que en El capital. 
En el otro extremo del espectro de los futuros imaginados del Antropoceno, hay ideas de un "buen Antropoceno", con la esperanza de formas aún más intensas del control humano de la naturaleza (Bai et al., 2016; Shellenberger y Nordhaus, 2015; Crutzen, 2006, Ellis, 2015).

En The Great Derangement, Ghosh (2016) trata el problema de la catástrofe desde el punto de vista de la ficción literaria y sus visiones uniformadoras del mundo, y deja a la ciencia ficción como género la posibilidad de ocuparse de los desastres provocados por la degradación. Me parece que su crítica a la vanguardia literaria ha fallado en reconocer que esa posibilidad sea convincente, aunque incómoda, pues las visiones uniformadoras del mundo no han prevalecido dentro de las ciencias y las humanidades. Por lo tanto, son cómplices de los autores de la "ficción seria" en la construcción del gran desajuste o desarreglo — derangement-.

Pero, ¿cómo se puede evitar el gran desajuste que termina en catástrofe? ¿Cómo puede revertirse la modernidad más allá del colapso? Las reflexiones de Ghosh sobre la historia y la política nos dejan sin ilusiones y con pocas esperanzas. Se trata de un análisis inflexible de las relaciones de poder en la política global actual, dominada por una angloesfera poco dispuesta a mitigar el cambio climático mediante la reducción significativa de emisiones de carbono. Su lectura del Acuerdo de París, así como la de la Encíclica Laudato Si, nos despoja de cualquier ilusión que tuviéramos al respecto. Eso nos deja con el dilema de cómo se pueden revertir las tendencias potencialmente catastróficas de la modernidad — calentamiento global, extinción de especies, etcétera-.

Entre las ideas para lograr esto, se encuentran desincentivar las inversiones y el decrecimiento, que recientemente han ganado alguna atención. Si bien las estrategias para internalizar los costos de seguimiento externos de las emisiones de carbono por medio de la fijación de precios - certificados de emisión, en otras palabras, la estrategia de Kyoto- han perdido crédito, los mercados de energía están empezando a cambiar. Poderosos inversionistas privados han apoyado iniciativas para desincentivar la inversión al retirar enormes cantidades de capital de la rama de combustibles fósiles. Una parte del capital despojado ha sido —o será- reinvertido en el sector de la energía sustentable emergente, que traerá de vuelta — jotra inversión! - nuestros sistemas de energía a los recursos locales y regionales: recordemos que el viento, el agua y la densidad de radiación solar dependen de la geografía local. Al llegar a un nivel crítico, la liquidación y reinversión podrían convertir los mercados de capitales en un acelerador del siguiente acto schumpeteriano de "destrucción creativa", del que es probable que surja rápidamente, tal vez incluso en cuestión de décadas, un nuevo régimen energético. En cualquier caso: ¡la energía es la clave! No es la solución a todos los problemas ambientales creados por el industrialismo fósil, pero es el problema más urgente, ya que ninguno de los otros se resolverá sin un desacoplamiento exitoso de los regímenes de energía de las emisiones de gases de efecto invernadero. Las trayectorias dependientes que han surgido con el régimen de fósiles están resistiendo estos cambios: acumulación de capital que se necesitaba para meterse de lleno; grandes empresas que ofrecen centrales eléctricas; redes centralizadas para la distribución de energía eléctrica; Estados centralizados y fuertes que garantizaban estabilidad para las inversiones de gran capital por medio de sus bancos nacionales y sistemas jurídicos. La transformación de nuestros sistemas de energía transformará nuestras sociedades enteras. ¡En todos lados! ${ }^{19}$

19

Las implicaciones, para la democracia, de cambiar el régimen de energía fósil de carbón a petróleo han sido recientemente exploradas por Mitchell (2013). Ian Morris et al. (2015) también han hecho un intento raro pero controvertido al considerar la historia profunda de los sistemas de valores desde la perspectiva de los regímenes energéticos. La obra de Vaclav Smil también merece especial atención. en particular Smil (2008). 
La reversión de las expectativas modernas de crecimiento económico eterno plantea un problema aún mayor. Esto es, por supuesto, si se espera que el decrecimiento tenga lugar como un proceso organizado y no como resultado de un colapso catastrófico. Las discusiones sobre el decrecimiento son notables por sus visiones de estilos de vida futuros, que ya no dependen de la expansión de nuestra participación en la riqueza material como individuos o sociedades, pero carecen de realismo sobre las trayectorias dependientes que las economías de crecimiento han creado en contra de dejarlos atrás. La realidad de la economía keynesiana —el New Deal - ha dado lugar a una acumulación de la deuda al hacer que nuestras economías dependan aún más del crecimiento futuro. El decrecimiento dejaría a las generaciones futuras sin la opción de usar la inflación para saldar la deuda. Por lo tanto, está claramente en falta de armonía con la justicia intergeneracional. ¿Y cómo podría el decrecimiento evitar alguna vez la austeridad y las consecuencias que han tenido hasta el momento la consolidación de la desigualdad y el poder social? Si, por el contrario, el decrecimiento es la única manera de revertir las tendencias actuales de destrucción ambiental, entonces la economía New Deal ya no es una opción.
El razonamiento sobre el destino de la modernidad ha sido una especie de ejercicio académico regular en las últimas décadas, quizá el debate sobre la posmodernidad sea la madre de todos. Pero el Antropoceno es algo nuevo bajo el sol; aunque no lo es si la idea es confundida con otra versión del viejo y familiar diagnóstico de una crisis ambiental que debe ser contada entre las consecuencias de la modernidad, como si pudiese ser tratada en un capítulo complementario del famoso libro de Anthony Giddens (1990). Clive Hamilton (2016) y otros han argumentado de manera convincente que eso es un malentendido. Reconocer que la humanidad se ha convertido en el equivalente a una fuerza geológica es un cambio gestalt en el pensamiento ecológico. Esto significa que la historia humana interfiere con escalas de tiempo geológicas, por ejemplo, al afectar el ciclo de carbono por medio de las emisiones de la quema de combustibles fósiles. Aunque el cambio global antropogénico es de hecho una consecuencia de la relación explotadora de la modernidad con la naturaleza, la idea del Antropoceno pide más de un capítulo en la historia de la modernidad o del capitalismo mismo. Cambia toda la historia (Chakrabarty, 2009; 2011; 2015; Mauelshagen, 2012). D

\section{Bibliografía}

Bai, Xuemei et al., 2016, "Plausible and Desirable Futures in the Anthropocene: A New Research Agenda”, en Global Environmental Change, núm. 39, pp. 351-362.

Bonneuil, Christophe y Jean-Baptiste Fressoz, 2016, The Shock of the Anthropocene: The Earth, History, and Us, Verso Books, Nueva York.

Chakrabarty, Dipesh, 2009, "The Climate of History: Four Theses”, en Critical Inquiry, vol. 35, núm. 2, pp. 197-222.

—_, 2011, “Verändert der Klimawandel die Geschichtsschreibung?”, en Transit. Europäische Revue, vol. 41, pp. 143-163.

__. 2014, "Climate and Capital: On Conjoined Histories”, en Critical Inquiry, vol. 41, núm. 1, pp. 1-23.

_ 2015, "The Anthropocene and the Convergence of Histories", en Clive Hamilton, Christophe Bonneuil y François Gemenne (eds.), The Anthropocene and the Global Environmental Crisis: Rethinking Modernity in a New Epoch, Routledge, Londres y Nueva York, pp. 44-56.

Cochet, Yves, 2015, "Gree Eschatology”, en Clive Hamilton, Christophe Bonneuil y François Gemenne (eds.), The Anthropocene and the Global Environmental Crisis, Routledge, Londres y Nueva York, pp. 112-120.

Crutzen, Paul J., 2002a, “The 'Anthropocene'”, en Journal de Physique IV (Proceedings), vol. 12, núm. 10, pp. 1-5.

— 2002b, "Geology of Mankind”, en Nature, vol. 415, núm. 6867, p. 23. 
_ 2006, "Albedo Enhancement by Stratospheric Sulfur Injections: A Contribution to Resolve a Policy Dilemma?”, en Climatic Change, núm. 77, pp. 211-220.

Crutzen, Paul J. y Eugene F. Stoermer, 2000, "The 'Anthropocene”, en Global Change Newsletter, núm. 41, pp. 17-18.

Descola, Philippe, 2013, Beyond Nature and Culture, The University of Chicago Press, Chicago y Londres.

Durkheim, Émile, 1897, Le suicide, Félix Alcan, París.

, 1982, The Rules of Sociological Method, Free Press, Nueva York.

Ellis, Erle, 2015, "Die gebrauchte Erde. Der Mensch als Gestalter der Erdgeschichte”, en Nina Möllers, Christian Schwägerl y Helmuth Trischler (eds.), Willkommen im Anthropozän. Unsere Verantwortung für die Zukunft der Erde, Deutsches Museum Verlag, Múnich, pp. $52-55$.

Ghosh, Amitav, 2016, The Great Derangement: Climate Change and the Unthinkable, University of Chicago Press, Chicago.

Giddens, Anthony, 1990, The Consequences of Modernity, Polity Press/Blackwell, Cambridge.

Hamilton, Clive, 2016, "The Anthropocene as Rupture", en The Anthropocene Review, vol. 3, núm. 2, pp. 93-106.

Haraway, Donna, 2015, “Anthropocene, Capitalocene, Plantationocene, Chthulucene: Making Kin”, en Environmental Humanities, núm. 6, pp. 159-165. Jefferson, Thomas, 1958, Papers of Thomas Jefferson, Princeton University Press, Princeton.

2016, "Thomas Jefferson to James Madison, 6 september, 1789", en Founders Online. Disponible en línea: <http://founders.archives.gov/ documents/Jefferson/01-15-02-0375-0003>. Consultado el 4 de septiembre de 2016

Kagan, Jerome, 2009, The Three Cultures: Natural Sciences, Social Sciences, and the Humanities in the $21^{5 t}$ Century, Cambridge University Press, Cambridge y Nueva York.

Latour, Bruno, 2005, Reassembling the Social. An Introduction to Actor-Network-Theory, Oxford University Press, Oxford.

_ 2013, An Inquiry into Modes of Existence: An Anthropology of the Moderns, Harvard University Press, Cambridge.

__ , 2015, Face à Gaïa: huit conférences sur le nouveau régime climatique, Les empêcheurs de penser en rond/La Découverte, París.

Lewis, Simon L. y Mark A. Maslin, 2015, "Defining the Anthropocene”, en Nature, vol. 519, núm. 2, pp. 128-146.

Marsh, George P., 1877, The Earth as Modified by Human Action: A New Edition of Man and Nature, Scribner/Armstrong, Nueva York.

Marx, Karl, 2000, Early Writings, Penguin, Londres.

Mauelshagen, Franz, 2012, “The 'Anthropocene': A Case for a Climate History of the Nineteenth and Twentieth Centuries”, en Zeithistorische Forschungen/Studies in Contemporary History, vol. 9, núm. 1. Disponible en línea: <http://www.zeithistorische-forschungen.de/1-2012/ $\mathrm{id}=4596$ \#en>.

_ 2015, "Defining Catastrophes", en Katharina Gerstenberger y Tanja Nusser (eds.), Catastrophe and Catharsis: Perspectives on Disaster and Redemption in German Culture and Beyond, Camden House, Rochester, pp. 172-190.

McNeill, John Robert y Peter Engelke, 2014, The Great Acceleration: An Environmental History of the Anthropocene since 1945, The Belknap Press of Harvard University Press, Cambridge.

Mitchell, Timothy, 2013, Carbon Democracy: Political Power in the Age of Oil, Verso Books, Londres.

Moore, Jason W., 2014a, "The Capitalocene. Part I: On the Nature and Origins of Our Ecological Crisis". Disponible en línea: <http://www. jasonwmoore.com/uploads/The_Capitalocene_Part_I_June_2014.pdf>. Consultado el 23 noviembre 2016.

_ _ 2014b, "The Capitalocene. Part II: Abstract Social Nature and the Limits to Capital”. Disponible en línea: <http://www.jasonwmoore. com/uploads/The_Capitalocene__Part_II_June_2014.pdf>. Consultado el 23 noviembre 2016.

_ (ed.), 2016, Anthropocene or Capitalocene? Nature, History, and the Crisis of Capitalism, PM Press, Oakland. Disponible en línea: <https://www.academia.edu/24341220/Anthropocene_or_Capitalocene_Nature_History_and_the_Crisis_of_Capitalism>. Consultado el 23 noviembre 2016.

Morris, lan et al., 2015, Foragers, Farmers, and Fossil Fuels: How Human Values Evolve, Princeton University Press, Princeton.

Northcott, Michael, 2015, "Eschatology in the Anthropocene: From the Chronos of Deep Time to the Kairos of the Age of Humans", en Clive Hamilton, Christophe Bonneuil y François Gemenne (eds.), The Anthropocene and the Global Environmental Crisis, Routledge, Londres y Nueva York, pp. 100-111.

Oreskes, Naomi y Erik M. Conway, 2014, The Collapse of Western Civilization: A View from the Future, Columbia University Press, Nueva York. Passerin d'Entrèves, Maurizio (ed.), 1996, Habermas and the Unfinished Project of Modernity: Critical Essays on the Philosophical Discourse of Modernity, en Polity Press, Cambridge.

Semal, Luc, 2015, "Anthropocene, Catastrophism and Green Political Theory”, en Clive Hamilton, Christophe Bonneuil y François Gemenne (eds.), The Anthropocene and the Global Environmental Crisis, Routledge, Londres y Nueva York, pp. 87-99.

Shellenberger, Michael y Ted Nordhaus, 2015, "An Ecomodernist Manifesto", en The Break Throug Journal, 15 de abril. Disponible en línea: <http://thebreakthrough.org/index.php/voices/michael-shellenberger-and-ted-nordhaus/an>. 
Smil, Vaclav, 2008, Energy in Nature and Society: General Energetics of Complex Systems, MIT Press, Cambridge.

Snow, C. P., 1959, The Two Cultures and the Scientific Revolution, Cambridge University Press, Nueva York.

Steffen, Will, Wendy Broadgate, Lisa Deutsch, Owen Gaffney y Cornelia Ludwig, 2015, "The Trajectory of the Anthropocene: The Great Acceleration”, en The Anthropocene Review, vol. 2, núm. 1, pp. 81-98.

Steffen, Will, Paul J. Crutzen y John R. McNeill, 2007, “The Anthropocene: Are Humans Now Overwhelming the Great Forces of Nature?”, en Ambio, vol. 36, núm. 8, pp. 614-621.

Steffen, Will, Jacques Grinevald, Paul Crutzen y John McNeill, 2011, “The Anthropocene: Conceptual and Historical Perspectives”, en Philosophical Transactions A, vol. 369, núm. 1938, pp. 842-867.

Waters, Colin N. et al., 2016, “The Anthropocene is Functionally and Stratigraphically Distinct from the Holocene”, en Science, vol. 351, núm. 6269. Zalasiewicz, Jan, 2010, The Planet in a Pebble: A Journey into Earth's Deep History, Oxford University Press, Oxford.

Zalasiewicz, Jan et al., 2015, "When did the Anthropocene Begin? A Mid-Twentieth Century Boundary Level is Stratigraphically Optimal”, en Quaternary International, vol. 383, pp. 196-203.

Zalasiewicz, Jan y Mark Williams, 2011, "The Anthropocene Ocean in its Deep Time Context”, en Davor Vidas y Peter Johan Schei (eds.), The World Ocean in Globalisation, Brill, Leiden, pp. 19-35.

Zalasiewicz, Jan, Mark Williams, Will Steffen y Paul Crutzen, 2010, “The New World of the Anthropocene", en Environmental Science and Technology, vol. 44, núm. 7, pp. 2228-2231. 